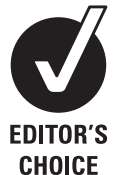

Cardiology Department, Royal Hallamshire Hospital, Sheffield, UK

Correspondence to Professor Kevin S Channer, Cardiology Department, Royal Hallamshire Hospital, Sheffield S10 2JF, UK

kevin.channer@sth.nhs.uk

Received 2 March 2010

Accepted 8 October 2010

Published Online First

19 November 2010

\title{
Reversible heart failure: the role of inflammatory activation
}

\author{
Tim Robinson, Alyson Smith, Kevin S Channer
}

\section{ABSTRACT}

Heart failure is increasingly common in western populations and is an inevitable consequence of the improved survival after myocardial infarction, and of an ageing population. Heart failure is usually relentlessly progressive as the maladaptive processes triggered by the physiological changes of the condition lead to further deterioration. However, in certain circumstances, heart failure is transient or potentially reversible when it occurs as part of intense systemic inflammatory activation. This review considers the role of inflammation in the aetiology of heart failure, and illustrates the strategies which have been used to modify the inflammatory response with anonymised clinical case reports.

\section{INTRODUCTION}

Chronic heart failure (CHF) is a major health burden in the western world, affecting about $2 \%$ of the population. The prevalence increases with age from about $1 \%$ in the fourth decade to over $10 \%$ in the seventh decade. It is now the most common cause of hospital admission in individuals over 65 years old. ${ }^{1}$ The syndrome is characterised by the inability of the heart to maintain the circulation, resulting in shortness of breath, fluid retention, and eventually cachexia. In the vast majority of cases, CHF is a relentlessly progressive condition with a poor prognosis-worse than many cancers. Follow-up studies show that $50 \%$ survival from first presentation with CHF is only about 5 years. ${ }^{2}$ Progression occurs because of the physiological maladaptations that develop in CHF, resulting in over expression of neurohormones such as norepinephrine and angiotensin II, as a result of activation of the sympathetic nervous system and the renin-angiotensin system. ${ }^{3}$ Drug treatments are aimed at interrupting the pathophysiology. Symptom palliation and extension of life have been demonstrated with drugs including angiotensin converting enzyme (ACE) inhibitors, aldosterone antagonists, and $\beta$-blockers, alone and in combination. ${ }^{4}$ Both patients with ischaemic and nonischaemic causes of CHF can present precipitously with fulminant CHF. However, progression of CHF depends upon the aetiology-patients with ischaemic heart failure have a worse prognosis than those with a non-ischaemic aetiology. 5 The reason for this is that ischaemic events recur causing stepwise deterioration in heart function, whereas progression in those patients with heart failure as a result of a single toxic insult tends to be much slower. However, it is rare to find patients who actually improve and in whom heart function objectively returns to normal. It is this group of patients with reversible heart failure that is the focus of this review.

\section{INFLAMMATORY CONDITIONS}

Rheumatoid arthritis (RA) is the most common inflammatory arthritis, affecting $1 \%$ of the general population. ${ }^{6}$ The clinical course is variable and the long term prognosis is poor, with $80 \%$ of patients disabled after 20 years. The pathophysiology of $\mathrm{RA}$ is centred on the synovium, which becomes hyperplastic, with increased vascularity and infiltration of inflammatory cells. The inflammatory cells are primarily CD4 $+\mathrm{T}$ cells which when activated stimulate macrophages, monocytes, and synovial fibroblasts to produce inflammatory cytokines-tumour necrosis factor $\alpha$ (TNF $\alpha)$, interleukin 1 (IL-1) and IL-6-and secrete matrix metalloproteinases. The $\mathrm{CD} 4+\mathrm{T}$ cells also stimulate $\mathrm{B}$ cells to produce immunoglobulins, such as rheumatoid factor. ${ }^{6} \mathrm{RA}$ is associated with increased cardiovascular morbidity ${ }^{7}$ and mortality, ${ }^{78}$ accounting for $30-50 \%$ of all RA related deaths. ${ }^{9}$ Cardiovascular disorders associated with RA include pericarditis, myocardial infarction, and $\mathrm{CHF}^{10}$ Heart failure is almost twice as common even after correction for ischaemic heart disease and cardiovascular risk factors in long term studies. ${ }^{11} \mathrm{CHF}$ is more common in patients with RA (3.9\%) compared to those with osteoarthritis (2.3\%). ${ }^{12}$ Reasons for this increased cardiovascular risk have been postulated to be due to vascular inflammation resulting in atherosclerosis, dyslipidaemia, hyperhomocysteinaemia, and reduced exercise capacity. In relation to $\mathrm{CHF}$ risk there is a direct suppressive effect on the myocardium of the inflammatory cytokines TNFa and IL-1.

The effect of drug induced immune modulation on CHF in patients with RA has shown that CHF was significantly less common in patients treated with anti-TNF drugs (3.1\% vs 3.8\%, p<0.05), after adjustment for baseline differences. ${ }^{12}$ In another study, patients treated with TNF $\alpha$ antagonists had only a $50 \%$ chance of developing heart failure sufficiently severe to merit hospitalisation, with a $20 \%$ reduction seen in those treated with methotrexate as monotherapy. ${ }^{13}$

\section{Illustrative case}

A middle aged man was admitted as an emergency with a 5 week history of increasing breathlessness, orthopnoea, paroxysmal nocturnal dyspnoea, and swelling of his ankles. He was a smoker, but had no other cardiovascular risk factors. He had previously been well, but had a 15 year history of intermittent joint pain affecting his hands, wrists, elbows, shoulders, ankles, and feet. Before admission his 
joint pains had become more severe and prolonged. On examination he had signs of heart failure, with a persistent sinus tachycardia, a raised jugular venous pressure wave, gallop rhythm, and bibasal crepitations. Examination of his joints revealed nodules over the proximal interphalangeal joints (PIJ) of the left hand and pain and restricted movement of the left elbow. Investigations showed haemoglobin $11.1 \mathrm{~g} / \mathrm{dl}$, raised $\mathrm{C}$ reactive protein (CRP) $203 \mathrm{mg} / 1$ (normal $<6 \mathrm{mg} / \mathrm{l}$ ), and rheumatoid factor $424 \mathrm{iu} / 1$ (normal $<20 \mathrm{iu} / \mathrm{l}$ ). All other blood tests were normal. An echocardiogram showed a dilated and severely impaired left ventricle, with calculated ejection fraction (EF) of $18 \%$, and left ventricular end diastolic dimension (LVEDD) of $70 \mathrm{~mm}$. He was treated with a loop diuretic, ACE inhibitor, $\beta$-blocker, and digoxin. He made a good recovery and was discharged home 10 days later. At subsequent review 6 weeks later, he complained of breathlessness on moderate exercise with no evidence of cardiac failure on examination. However, he had inflamed wrists and PIJs on both hands. He was started on prednisolone $5 \mathrm{mg}$ per day, and a subsequent review by a rheumatologist led to the diagnosis of $\mathrm{RA}$ and the addition of methotrexate to his treatment regimen. His arthritis symptoms responded well to treatment and his exercise tolerance normalised. An echocardiogram 6 months after presentation showed a non-dilated left ventricle with good function, EF $65 \%$, and LVEDD $55 \mathrm{~mm}$.

\section{Comment}

This man presented for the first time with acute RA with concomitant CHF. Heart failure resolved coincidentally with improvement in his active arthritis and objective assessment by echocardiography indicated that heart function had returned to normal. This case illustrates the temporary effect of high cytokine activation on myocardial function.

\section{CARDIOMYOPATHY OF INTENSIVE CARE}

The transient heart failure that occurs in patients with multiorgan dysfunction typically after multiple trauma or severe sepsis is also thought to be caused by acute cytokine activation. ${ }^{14}$ This group of patients requires multiorgan support and therefore tend to cluster in intensive care units (ICUs). Intensivists have recognised the transient heart failure seen in this situation and called it the 'cardiomyopathy of intensive care' or 'critical illness cardiomyopathy'. A number of serial echocardiographic studies have been done on patients with septic shock on ICUs showing that reversible ventricular dilatation and systolic dysfunction occurs in up to $60 \%$ of cases with no impact on prognosis. ${ }^{15}$ Bouhemad et al ${ }^{16}$ also found a correlation between the troponin blood value and concentrations of TNF $\alpha$, Il-8, and Il-10 and left ventricular systolic dysfunction (LVSD), which normalised coincidentally with falling cytokine concentrations. In another study of consecutive patients admitted to an ICU with septic shock without a previous history of underlying heart disease, the concentration of B-type natriuretic peptide (BNP) in the blood correlated with echocardiographic abnormalities of LVSD and prognosis. ${ }^{17}$

Not all patients with echocardiographic evidence of LVSD have positive troponin blood values, but when this does occur, some series suggest it is a marker of a worse prognosis. In a series of 128 consecutive admissions to a medical ICU, troponin elevation was seen in $54 \%$ cases of sepsis, $17 \%$ cases of respiratory failure, and $9 \%$ of poisonings. However, troponin release is more likely to occur in patients with underlying ischaemic heart disease and it is not clear whether these patients had known underlying heart disease. ${ }^{18}$

\section{Illustrative case}

A woman with a long history of Crohn's disease had a normal twin pregnancy and delivered vaginally at 32 weeks gestation. Three weeks later she developed an acute colitis, with pronounced inflammatory activation resulting in toxic dilatation, which necessitated an emergency total colectomy 2 weeks later. She had a stormy postoperative course complicated by gram negative sepsis, and 10 days after surgery she was admitted to the ICU because of increasing breathlessness, tachycardia, and hypertension. She was found to have pulmonary hypertension (pulmonary artery pressure $44 / 29 \mathrm{~mm} \mathrm{Hg}$ ) and initially mild then more severe left ventricular dysfunction (EF 30\% on echocardiography). Two weeks later cardiac catheterisation revealed normal coronary arteries, severe global LVSD (EF 30\%), and low right sided pressures. She responded to $\beta$-adrenoceptor blockade and vasodilators including an ACE inhibitor and improved clinically. Echocardiography showed EF had increased to $50 \%$ by 4 months, and by 8 months the appearances were normal (EF 67\%).

\section{Comment}

In this case the CHF was thought to be secondary to the flare-up of the patient's Crohn's disease, gram negative sepsis, and the subsequent insult of major abdominal surgery, although peripartum cardiomyopathy (PPCM) could not be excluded.

\section{PERIPARTUM CARDIOMYOPATHY}

PPCM is a special form of cardiomyopathy which is a distinct condition, rather than worsening of pre-existing heart failure. It is defined by four criteria: (1) the development of cardiac failure in the last month of pregnancy or within 5 months of delivery; (2) absence of an identifiable cause for heart failure; (3) absence of recognisable heart disease before the last month of pregnancy; and (4) LVSD. Predisposing factors reported include increased maternal age, African origin, use of tocolytics, multiparity, multiple pregnancy, and pre-eclampsia. ${ }^{19}$

Early signs and symptoms of heart failure, especially breathlessness and ankle oedema, are often attributed to normal pregnancy. Left ventricular thrombus is common in patients with PPCM, especially with an EF $<35 \%$, as pregnancy is a prothrombotic state. This may lead to peripheral and organ embolisation. About $50-60 \%$ of patients with PPCM recover completely with normalisation of left ventricular systolic function. It has been shown that failure to regain this function at 6 months after pregnancy is an indicator of permanent LVSD. Some echocardiographic parameters predict improvement (higher EF and smaller left ventricular dimensions). ${ }^{19}$

The underlying pathogenesis of PPCM remains unknown, although the clinical behaviour and natural history suggests an inflammatory aetiology perhaps with an autoimmune basis. ${ }^{20}$ Melvin et $a l^{21}$ found dense lymphocytic infiltrates in cardiac biopsies from patients with PPCM, and subsequent treatment with immunosuppressive drugs resulted in improvement of left ventricular function and loss of the inflammatory infiltrate. Sliwa and colleagues ${ }^{22}$ identified increased concentrations in plasma of the inflammatory cytokine TNF $\alpha$, CRP, and a plasma marker of apoptosis, Fas/Apo-1, in a large population of newly diagnosed patients with PPCM. Baseline concentrations of CRP correlated positively with baseline left ventricular end-diastolic and end-systolic diameters and inversely with left ventricular EF. Concentrations of Fas/Apo-1 in plasma were significantly higher in patients with PPCM than in healthy controls, and were a predictor of mortality.

Immunoglobulin has been used in the treatment of postpartum cardiomyopathy with encouraging trial results. ${ }^{23}$ Drexler et al 
demonstrated that a biologically active derivative of prolactin mediates PPCM, and that inhibition of prolactin release with bromocriptine may represent a novel therapeutic option. ${ }^{24}$

Amniotic fluid embolisation causes a similar pattern of cytokine activation with multiorgan dysfunction. This is rare, occurring in 1 in 8000-80000 pregnancies, and results in acute haemodynamic collapse, akin to an anaphylactoid reaction caused by profound inflammatory, vasospastic, and pro-coagulatory activation. $^{25}$

\section{Illustrative case}

A Caucasian woman had a normal pregnancy complicated in the last few weeks by mild hypertension (untreated), and delivered normally at term. About $12 \mathrm{~h}$ later she developed breathlessness and chest tightness. Examination revealed a sinus tachycardia of 110 beats/min, blood pressure was $100 / 60 \mathrm{~mm} \mathrm{Hg}$, and the central venous pressure was elevated to $4 \mathrm{~cm}$. There were bi-basal crackles in the lung bases. A chest x-ray showed acute pulmonary oedema. She was treated with intravenous furosemide and she rapidly improved. The following day an echocardiogram showed a dilated and moderately impaired left ventricle with an estimated EF of $35 \%$ and LVEDD of $6.2 \mathrm{~cm}$. She was treated with an ACE inhibitor and a low dose $\beta$-blocker was started. By 3 months she no longer needed the diuretic, she was taking full doses of an ACE inhibitor and $\beta$-blocker, and her effort tolerance had returned to normal. By 6 months the echocardiographic appearances showed a notable improvement, with mild hypokinesia of the interventricular septum (EF 60\% and LVEDD $5.4 \mathrm{~cm}$ ). Three years later she remains well with normal effort tolerance.

\section{VIRAL CARDIOMYOPATHY}

Patients with acute viral infections may develop myocarditis, the natural history of which is very variable, resulting in acute fulminant, subacute or chronic myocarditis and chronic persistent myocarditis. Many viruses having been implicated in the pathogenesis of infectious myocarditis, and often the actual virus is not identified as in this case.

Viral myocarditis causes an initial non-specific acute inflammatory activation including natural killer cells, cytokines, interferon, TNF, interleukins, and proteins such as perforins and apolipoprotein $J$. The initial response is followed by activation of CD4 T lymphocytes and release of cytokines like interferon $\gamma$, TNF, IL-2, IL-3, IL-4, IL-5, IL-6, and IL-10. Immunologic sequences of events ultimately lead to production of CD4 and CD8 $\mathrm{T}$ lymphocytes and IgG antibodies specific for the virus. It has been suggested that coxsackie virus may have an antigen similar to myosin, which may result in long term autoimmune attack. Viral infections also lead to stimulation of interferon $\alpha$ and $\gamma$, which induce nitric oxide synthase (NOS), leading to increased concentrations of nitric oxide which in turn suppresses viral replication and prevents myocardial dysfunction. However, excess NOS can result in production of free radicals that can cause cell death, depressing myocardial function. In a study by Fairbrother et $a l^{26}$ an association was found between complement receptor 1 and 2 deficiencies, with acute coxsackie B3 myocarditis. Other immunological responses such as expression of Toll-like receptor 4, upregulation of the cysteine-rich protein gene (cyr61), and activation of transcription factor $\kappa \mathrm{B}$ have been implicated in the inflammatory response of viral myocarditis, which may contribute to cell death and myocardial dysfunction. ${ }^{27-29}$ Finally, myocyte necrosis and cardiac dilatation may result from coronary microvascular spasm. ${ }^{30}$ Endothelium dysfunction resulting from endothelial cell antibodies may also contribute to this microvascular spasm. ${ }^{31}$

\section{Illustrative case}

A previously fit woman was admitted with increasing effort intolerance following a prodromal flu-like syndrome with myalgia and headache. She was a non-smoker and consumed 10 units of alcohol per week. She had no significant past medical history or any other cardiovascular risk factors. Apart from atrial fibrillation there were no other overt signs of heart failure. Electrocardiography, and subsequent Holter monitoring, confirmed atrial fibrillation with a controlled ventricular rate, and routine blood tests including thyroid function and ferritin were normal. An echocardiogram showed a dilated left ventricle (LVEDD $61 \mathrm{~mm}$ ) with an EF of $34 \%$. There was mild functional mitral regurgitation and slight enlargement of the left atrium $(42 \mathrm{~mm}$, normal $<40 \mathrm{~mm}$ ). During admission, the patient developed acute left ventricular failure and was treated with loop diuretics, ACE inhibitors, and warfarin. She recovered completely and was discharged 2 weeks later. Paired serological tests for coxsackie A and $\mathrm{B}, \mathrm{ECHO}$ (enteric cytopathic human orphan) virus and other enteroviruses, were negative. After 8 months, she had normal exercise capacity and the echocardiogram showed normal left ventricular and left atrial dimensions with an EF of $67 \%$.

\section{MODIFYING THE INFLAMMATORY RESPONSE IN HEART FAILURE}

We have described four clinical situations where pronounced inflammatory activation appears to be aetiologically linked with the development of heart failure. However, inflammatory activation is a feature of the syndrome of heart failure per se, and patients with CHF who have raised concentrations of inflammatory mediators ${ }^{32}{ }^{33}$ have a worse prognosis. ${ }^{34-39}$ Therefore, the use of anti-inflammatory therapies as a potential treatment target for patients with CHF has been studied. Two main anticytokine treatments have been tested: etanercept, given by subcutaneous injection, is a recombinant TNF receptor which binds TNF $\alpha$ and therefore prevents it from binding to cell surface receptors; and infliximab, given intravenously, is a monoclonal antibody which binds to TNF $\alpha$, thereby neutralising it.

Etanercept was first studied in vitro ${ }^{39}$ and in vivo ${ }^{40}$ and was shown to reverse the negative inotropic effects caused by TNF $\alpha$. In a subsequent pilot clinical trial in patients with $\mathrm{CHF}^{41}$ etanercept treatment appeared to improve quality of life scores and ventricular function. The results from two larger multicentre clinical trials (RENAISSANCE and RECOVER) were combined (RENEWAL) and powered to show a reduction in the primary end point of all cause mortality and hospitalisation for $\mathrm{CHF}^{42}{ }^{43}$ However, the trials were stopped early as an interim analysis indicated that the primary end point would not be met. It has been suggested that the main reasons for this lack of benefit related to the doses of etanercept given. ${ }^{44}$ The unadjusted mortality rates for the etanercept group, $25 \mathrm{mg}$ once a week, and placebo (RECOVER) were $5.9 \%$ and $8.8 \%$, respectively, suggesting a reduction in total mortality by $30 \%$ in the etanercept group. ${ }^{42}$ The overall results (RENEWAL) were not sufficiently favourable to signify a clinically relevant benefit. ${ }^{43}$

Two doses of infliximab were studied in 150 patients in the ATTACH trial. ${ }^{45}$ This trial was terminated early due to an excess of deaths and hospital admissions in the higher dose group. Results showed plasma concentrations of infliximab to be much higher than expected, and the adverse risk was only seen in the higher dose group. It was noted that the left ventricular EF (LVEF) improved in the lower dose group.

The negative results of these three trials may be explained by incorrect dosing, ${ }^{44}$ but also by the fact that cytokines have 
many different effects, both advantageous and disadvantageous, on the myocardium, and by blocking certain cytokines the balance between them may be altered. ${ }^{46}$ Therefore other immune modulating therapies have been tried. Corticosteroids were used in the $1980 \mathrm{~s}^{47}$ and later intravenous immunoglobulin has been studied. Intravenous immunoglobulin has been proposed to work by neutralisation of autoantibodies and alteration of cytokine activity, although the precise method is unknown. ${ }^{48}$ It has been shown to increase LVEF and plasma anti-inflammatory mediators in a group of 47 patients with moderate CHF. ${ }^{49}$ Immune modulation therapy applies physiochemical stresses to an autologous blood sample, which is then injected back into the patient intramuscularly. The process results in down-regulation of proinflammatory cytokines and an up-regulation of antiinflammatory cytokines. ${ }^{50}$ A trial of immune modulation therapy in 73 patients with moderate CHF showed reductions in hospitalisations and death and improvements in New York Heart Association (NYHA) classification and quality of life. ${ }^{52}$

\section{WHEN TO CONSIDER INFLAMMATION IN THE AETIOLOGY OF HEART FAILURE?}

The clinical cases we have described were associated with known inflammatory conditions, but proof of active cardiac involvement in the inflammatory process is often difficult to find.

Endomyocardial biopsy (EMB) can provide a histological diagnosis in patients with initially unexplained cardiomyopathy. However, one study showed that a positive result was only obtained in $15.3 \%$ of patients, and with a complication rate of $8 \%,{ }^{53}$ the role of EMB thus remains unclear. Irrespective of aetiology, clinicians must treat the syndrome of heart failure, with $\beta$-blockade and ACE inhibitors being indicated for all patients with LVSD. In the absence of disease modifying agents for inflammatory cardiac activation, EMB has limited value. Current guidelines recommend $\mathrm{EMB}$ in patients with acute or fulminant CHF of unknown aetiology who deteriorate rapidly with ventricular arrhythmias and/or atrioventricular heart block, who are unresponsive to conventional therapy. In chronic

\section{Key references}

- Levy D, Kenchaiah S, Larson MG, et al. Long-term trends in the incidence of and survival with heart failure. $N$ Engl J Med 2002;347:1397-402.

- Mann DL, McMurray JJ, Packer M, et al. Targeted anticytokine therapy in patients with chronic heart failure: results of the Randomized Etanercept Worldwide Evaluation (RENEWAL). Circulation 2004;109:1594-602.

- Chung ES, Packer M, Lo KH, et al. Anti-TNF Therapy Against Congestive Heart Failure Investigators. Randomized, doubleblind, placebo-controlled, pilot trial of infliximab, a chimeric monoclonal antibody to tumour necrosis factor-alpha, in patients with moderate-to-severe heart failure: results of the anti-TNF Therapy Against Congestive Heart Failure (ATTACH) trial. Circulation 2003;107:3133-40.

- Felker GM, hompson RE, Hare JM, et al. Underlying causes and long-term survival in patients with initially unexplained cardiomyopathy. N Engl J Med 2000;342:1077-84.

- Dickstein K, Cohen-Solal A, Filippatos G, et al. ESC guidelines for the diagnosis and treatment of acute and chronic heart failure 2008. Eur Heart $J$ 2008;29:2388-442.

\section{Main messages}

Not all heart failure is relentlessly progressive.

- Acute inflammatory activation can cause transient suppression of myocardial function.

- Supportive treatment while the underlying disease process is corrected results in cardiac recovery.

- When chronic heart failure is associated with inflammatory activation, prognosis is worse.

\section{Current research questions}

- How is inflammatory activation in chronic heart failure identified?

- Can drug treatments manipulate inflammatory processes in heart failure to improve outcome?

CHF, EMB should be considered if an infiltrative process such as sarcoid, amyloid or haemochromatosis is suspected, as well as in eosinophilic myocarditis and restrictive cardiomyopathy of unknown origin. ${ }^{54}$

\section{CONCLUSIONS}

Although the management of heart failure does not significantly differ with respect to its aetiology, it is important to recognise the common reversible causes. This series of case reports illustrates how inflammatory activation can cause reversible LVSD. It illustrates the pivotal role of inflammatory activation and especially cytokine activation in the aetiology and progression of disease. Recognising and therefore managing these conditions appropriately can prevent irreversible loss of myocardial function. This may lead to optimal utilisation of resources, better control of symptoms, and prevent the increased mortality that is seen commonly with CHF. The utility of cardiac biopsy remains unclear and further data to support the usefulness of targeting pro-inflammatory mediators as a treatment option for CHF are required.

\section{MULTIPLE CHOICE QUESTIONS (TRUE (T)/FALSE (F); ANSWERS AFTER THE REFERENCES)}

1. Regarding rheumatoid arthritis and congestive heart failure (CHF):

A. $30-50 \%$ of rheumatoid arthritis-related deaths are associated with cardiovascular morbidity and mortality

B. CHF is less common in rheumatoid arthritis than in osteoarthritis

C. Tumour necrosis factor $\alpha$ (TNF $\alpha$ ), and interleukin 1 (IL-1) suppress the myocardial contractility

D. Immune modulation treatment in patients with rheumatoid arthritis with anti-TNF drugs increases the prevalence of CHF

\section{CHF in patients on an intensive care unit:}

A. Is caused by acute cytokine activation

B. Reversible ventricular dilatation and systolic impairment carry an adverse prognosis

C. Is associated with decreased concentrations of IL-8 and IL-10 
D. Elevated levels of serum troponin are commonly found with echocardiographic evidence of left ventricular systolic dysfunction

\section{Peripartum cardiomyopathy (PPCM):}

A. Is diagnosed if pre-existing $\mathrm{CHF}$ is exacerbated by pregnancy

B. Is more common in multi-parous women, in twin pregnancies and pre-eclampsia

C. Is often diagnosed late as symptoms and signs of heart failure may be attributed to changes seen in normal pregnancy

D. Can be treated with bromocriptine

\section{Viral myocarditis:}

A. Usually follows a predictable course

B. Causes an acute inflammatory activation of natural killer cells, interleukins, cytokines, TNF and interferon

C. Can follow a relapsing and remitting course

D. Is associated with reduced levels of nitric oxide synthase

\section{Regarding potential therapies for inflammatory causes of CHF:}

A. Etanercept and Infliximab are anti-cytokine treatments

B. Etanercept reverses myocardial suppression caused by TNF $\alpha$

C. Infliximab was associated with increased mortality at high doses

D. Immunoglobulin increases left ventricular ejection fraction

Competing interests None declared.

Contributors All authors provided cases and contributed to the reviewing process of literature and edited and approved the manuscript.

Provenance and peer review Not commissioned; externally peer reviewed.

\section{REFERENCES}

1. Lam CS, Donal E, Kraigher-Krainer E, et al. Epidemiology and clinical course of heart failure with preserved ejection fraction. Eur J Heart Fail Published Online First: Aug 2010. doi:10.1093/eurjhf/hfq121.

2. Levy D, Kenchaiah S, Larson MG, et al. Long-term trends in the incidence of and survival with heart failure. N Engl J Med 2002;347:1397-402.

3. Chaggar P, Malkin CJ, Shaw SM, et al. Neuro-endocrine effects on the heart and targets for therapeutic manipulation in heart failure. Cardiovascular Therapeutics 2009;27:187-93

4. Malkin C, Channer KS. Life-saving or life-prolonging? Interpreting trial data and survival curves for patients with congestive heart failure. European Journal of Heart Failure 2005; 7:143-8.

5. Steeds RP, Channer KS. Heart failure; vive la difference. Heart 1997;78:537-8.

6. Lee SS, Joo YS, Kim WU, et al. Vascular endothelial growth factor levels in the serum and synovial fluid of patients with rheumatoid arthritis. Clin Exp Rheumatol 2001:19:321-4

7. Wallberg-Jonsson S, Ohman ML, Dahlqvist SR. Cardiovascular morbidity and mortality in patients with seropositive rheumatoid arthritis in Northern Sweden. $J$ Rheumatol 1997:24:445-51.

8. Wolfe F, Mitchell DM, Sibley JT, et al. The mortality of rheumatoid arthritis. Arthritis Rheum 1994;37:481-94.

9. Reilly PA, Cosh JA, Maddison PJ, et al. Mortality and survival in rheumatoid arthritis: a 25 year prospective study of 100 patients. Ann Rheum Dis 1990;49:363-9.

10. Khan AH, Spodick DH. Rheumatoid heart disease. Semin Arthritis Rheum 1972:1:327-37.

11. Nicola PJ, Maradit-Kremers $\mathrm{H}$, Roger VL, et al. The risk of congestive heart failure in rheumatoid arthritis; a population-based study over 46 years. Arthritis Rheum 2005;52:412-20.

12. Wolfe F, Michaud K. Heart failure in rheumatoid arthritis; rates, predictors and the effect of anti-tumour necrosis factor therapy. Am J Med 2004:116:305-11.

13. Bernatsky S, Hudson M, Suissa S. Anti-rheumatic drug use and risk of hospitalisation for congestive heart failure in rheumatoid arthritis. Rheumatology (Oxford) 2005:44:677-80

14. Krishnagopalan S, Kumar A, Parrillo JE, et al. Myocardial dysfunction in the patient with sepsis. Curr Opin Crit Care 2002;8:376-88.

15. Vieillard-Baron A, Caille V, Charron $C$, et al. Actual incidence of global left ventricular hypokinesia in adult septic shock. Crit Care Med 2008;36:1701-6.

16. Bouhemad B, Nicolas-Robin A, Arbelot C, et al. Isolated and reversible impairment of ventricular relaxation in patients with septic shock. Crit Care Med 2008;36:766-74
17. Charpentier J, Luyt CE, Fulla Y, et al. Brain natiuretic peptide: a marker of myocardial dysfunction and prognosis during severe sepsis. Crit Care Med 2004:32:600-6.

18. King DA, Codish S, Novack V, et al. The role of cardiac troponin I as a prognosticato in critically ill medical patients: a prospective observational cohort study. Crit Care Med 2005:9:390-5.

19. Sutton MS, Cole P, Plappert $M$, et al. Effects of subsequent pregnancy on left ventricular function in peripartum cardiomyopathy. Am Heart $J$ 1991;121:1776-8.

20. Ansari AA, Neckelmann N, Wang YC, et al. Immunologic dialogue between cardiac myocytes, endothelial cells, and mononuclear cells. Clin Immunol Immunopathol 1993;68:208-14.

21. Melvin KR, Richardson PJ, Olsen EG, et al. Peripartum cardiomyopathy due to myocarditis. N Eng J Med 1982;307:731-4.

22. Sliwa K, Skudicky D, Bergemann A, et al. Peripartum cardiomyopathy; analysis of clinical outcome, left ventricle function, plasma levels of cytokines and Fas/APO-1. J Am Coll Cardiol 2000;35:701.

23. Bozkurt B, Villaneuva FS, Holubkov $\mathrm{R}$, et al. Intravenous immunoglobuluin in the therapy of peripartum cardiomyopathy. J Am Coll Cardiol 1999;34:177-80.

24. Hilfiker-Kleiner D, Kaminski K, Podewski E, et al. A cathepsin D-cleaved 16 kDa form of prolactin mediates postpartum cardiomyopathy. Cell 2007:128:589-600.

25. Moore J, Baldisseri MR. Amniotic fluid embolism. Crit Care Med 2005;33(10 Suppl):S279-85.

26. Fairbrother D, Frisanchio-Kiss S, Njoku DB, et al. Complement receptor 1 \& 2 deficincy increases coxsackie B3-induced myocarditis, dilated cardiomyopathy and heart failure by increasing macrophages, IL-1beta, and immune complex deposition in the heart. J Immun 2006;176:3516-24.

27. Alter $\mathbf{P}$, Rupp H, Maisch B. Activated nuclear transcription factor kappaB in patients with myocarditis and dilated cardiomyopathy-relation to inflammation and cardiac function. Biochem Biophys Res Commun 2006;339:180-7.

28. Satoh M, Nakamura M, Akatsu T, et al. Expression of toll-like receptor 4 is associated with enteroviral replication in human myocarditis. Clin Sci (Lond) 2003; 104:577-84.

29. Kim SM, Park JH, Chung SK, et al. Coxsackie virus B3 infection induces cyr61 activation via JNK to mediate cell death. J Virol 2004:78:13479-88.

30. Sole MJ, Liu PJ. Viral myocarditis: a paradigm for understanding the pathogenesis and treatment of dilated cardiomyopathy. J Am Coll Cardiol 1993;22(4 Suppl A):99A-105A.

31. Levine B, Kalman J, Mayer L, et al. Elevated circulating levels of tumour necrosis factor in severe heart failure. N Engl J Med 1990;323:236-41.

32. Testa M, Yeh M, Lee $P$, et al. Circulating levels of cytokines and their endogenous modulators in patients with mild to severe congestive heart failure due to coronary artery disease or hypertension. J Am Coll Cardiol 1996;28:964-71.

33. Torre-Amione G, Kapadia S, Benedict C, et al. Proinflammatory cytokine levels in patients with depressed left ventricular ejection fraction: a report from the Studies of Left Ventricular Dysfunction (SOLVD). J Am Coll Cardiol 1996;27:1201-6.

34. Ferrari R, Bachetti T, Confortini R, et al. Tumour necrosis factor soluble receptors in patients with various degrees of congestive heart failure. Circulation 1995:92:1479-86.

35. Tsutamoto T, Hisanaga T, Wada A, et al. Interleukin-6 spillover in the peripheral circulation increases with the severity of the heart failure, and the high plasma leve of interleukin-6 is an important prognostic predictor in patients with congestive heart failure. J Am Coll Cardiol 1998;31:391-8.

36. Seta $\mathbf{Y}$, Shan $\mathrm{K}$, Bozkurt B, et al. Basic mechanisms in heart failure: the cytokine hypothesis. J Card Fail 1996;2:243-9.

37. Rauchhaus $\mathbf{M}$, Doehner W, Francis DP, et al. Plasma cytokine parameters and mortality in patients with chronic heart failure. Circulation 2000;102:3060-7.

38. Deswal A, Petersen NJ, Feldman AM, et al. Cytokines and cytokine receptors in advanced heart failure: an analysis of the cytokine database from the Vesnarinone trial (VEST). Circulation 2001;103:2055-9.

39. Kapadia S, Torre-Amione G, Yokoyama T, et al. Soluble TNF binding proteins modulate the negative inotropic properties of the TNF-alpha in vitro. Am J Physio 1995;268:H517-25

40. Bozkurt B, Kribbs SB, Clubb FJ Jr, et al. Pathophysiologically relevant concentrations of tumour necrosis factor-alpha promote progressive left ventricular dysfunction and remodelling in rats. Circulation 1998;97:1382-91.

41. Deswal A, Bozkurt B, Seta Y, et al. Safety and efficacy of a soluble P75 tumour necrosis factor receptor (Enbrel, etanercept) in patients with advanced heart failure. Circulation 1999;99:3224-6.

42. Bozkurt B, Torre-Amione G, Warren MS, et al. Results of targeted anti-tumour necrosis factor therapy with etanercept (ENBREL) in patients with advanced heart failure. Circulation 2001;103:1044-7.

43. Mann DL, McMurray JJ, Packer M, et al. Targeted anticytokine therapy in patients with chronic heart failure: results of the Randomized Etanercept Worldwide Evaluation (RENEWAL). Circulation 2004;109:1594-602.

44. Anker SD, Coats AJ. How to RECOVER from the RENAISSANCE? The significance of the results of the RECOVER, RENAISSANCE, RENEWAL and ATTACH. Int $J$ Cardiol 2002;86:123-30

45. Chung ES, Packer M, Lo KH, et al; Anti-TNF Therapy Against Congestive Heart Failure Investigators. Randomized, double-blind, placebo-controlled, pilot trial of infliximab, a chimeric monoclonal antibody to tumour necrosis factor-alpha, in patients with moderate-to-severe heart failure: results of the anti-TNF Therapy Against Congestive Heart Failure (ATTACH) trial. Circulation 2003;107:3133-40. 
46. Henriksen PA, Newby DE. Therapeutic inhibition of tumour necrosis factor alpha in patients with heart failure: cooling an inflamed heart. Heart 2003;89:14-18.

47. Parrillo JE, Cunnion RE, Epstein SE, et al. A prospective, randomized, controlled trial of prednisone for dilated cardiomyopathy. N Engl J Med 1989;321:1061-8.

48. Samuelsson A, Towers TL, Ravetch JV. Anti-inflammatory activity of IVIG mediated through the inhibitory Fc receptor. Science 2001:291:484-6.

49. Gullestad L, Aass H, Fjeld JG, et al. Immunomodulating therapy with intravenous immunoglobulin in patients with chronic heart failure. Circulation 2001;103:220-5

50. Fadok VA, Bratton DL, Konowal A, et al. Macrophages that have ingested apoptotic cells in vitro inhibit proinflammatory cytokine production through autocrine/paracrine mechanisms involving TGF-beta, PGE2, and PAF. J Clin Invest 1998;101:890-8.

51. Voll RE, Roth EA, Girkontaite I, et al. Histone-specific Th0 AND Th1 clones derived from systemic lupus erythematosus patienrs induce double-stranded DNA antibody production. Arthritis Rheum 1997:40:2162-71.

52. Torre-Amione G, Sestier F, Radovancevic B, et al. Effects of a novel immune modulation therapy in patients with advanced chronic heart failure: results of a randomized, controlled, phase II trial J Am Coll Cardiol 2004:44:1181-6.
53. Felker GM, Thompson RE, Hare JM, et al. Underlying causes and long-term survival in patients with initially unexplained cardiomyopathy. $N$ Engl J Med 2000:342:1077-84.

54. Dickstein K, Cohen-Solal A, Filippatos G, et al. ESC guidelines for the diagnosis and treatment of acute and chronic heart failure 2008. Eur Heart J 2008;29 $2388-442$

\section{ANSWERS}

1. $A(T) ; B(F) ; C(T)$; $D(F)$

2. $A(T) ; B(F) ; C(F) ; D(T)$

3. $A(F)$ : $B(T) ; C(T) ; D(T)$

4. $A(F) ; B(T) ; C(T) ; D(F)$

5. $A(T) ; B(T) ; C(T) ; D(T)$ 